\title{
L'approche de formation hybride comme méthode d'enseignement facilitant la compréhension en lecture d'un cours de spécialité dispensé en FLE au supérieur algérien
}

The hybrid training approach as a teaching method that facilitates the reading comprehension of a speciality course given in French as a foreign language class at Algerian higher education level

El enfoque de la formación híbrida como método de enseñanza que facilita la comprensión lectora de una especialidad impartida en francés como lengua extranjera en la enseñanza superior argelina

Amira Khelfi, doctorante

LISEC, Université Haute Alsace Mulhouse, France amira.khelfi@uha.fr

Sondess Zarrouk-Ben Abid, docteur HDR

LISEC, Université Haute Alsace Mulhouse, France sondess.zarrouk@uha.fr 
Notre problématique de recherche est née d'un double constat établi à partir d'une observation personnelle faite auprès d'apprenants arabophones confrontés à la compréhension en lecture des contenus pédagogiques, qui au travers de nos lectures a été davantage consolidée, notamment avec les travaux de Boudechiche (2008), Sebane (2008) et Rekrak (2016) dans lesquels ils ont démontrés avec clarté que les étudiants, en tentant de construire des connaissances en français langue étrangère, rencontraient beaucoup de difficultés. Nous supposons que l'exploitation des multiples apports d'une plateforme d'apprentissage en ligne "MoodleCloud" dans le cadre d'un enseignement hybride permettrait d'enrichir l'enseignement dispensé en classe et de développer au mieux la compétence de compréhension en lecture de ces étudiants. Afin de vérifier nos hypothèses de recherche, nous avons mené tout au long d'un semestre d'étude une enquête sur terrain qui s'est déroulée en trois phases auprès d'étudiants algériens inscrits en première année Licence au département de Français.

Mots-clés : dispositif de formation hybride, compréhension en lecture, français langue étrangère, pédagogie universitaire, contexte universitaire algérien

\section{ABSTRACT}

Our research problem arose from a twofold observation based on personal observation of Arabic-speaking learners confronted with reading comprehension of pedagogical content, which through our reading has been further consolidated, notably with the work of Boudechiche (2008), Sebane (2008) and Rekrak (2016) in which they clearly demonstrated that students, in trying to build knowledge in French as a foreign language, encounter many difficulties. We assume that using the multiple contributions of a "MoodleCloud" e-learning platform in a hybrid education approach would enrich classroom instruction and best develop the reading comprehension skills of these students. In order to verify our research hypotheses, we conducted a three-phase field survey throughout a semester of study with Algerian students enrolled in the first year of their bachelor's degree in the French department.

Keywords: hybrid training device, reading comprehension, French as a foreign language, university pedagogy, Algerian university context

\section{RESUMEN}

Nuestro problema de investigación surgió a partir de una observación personal, realizada con alumnos de lengua árabe confrontados a la comprensión lectora de contenidos pedagógicos, que se ha consolidado aún más a través de nuestras lecturas, en particular con los trabajos de Boudechiche (2008), Sebane (2008) y Rekrak (2016), en los que demostraron claramente que los estudiantes se encuentran con muchas dificultades al tratar de construir conocimiento del francés como lengua extranjera.. Suponemos que el uso de las múltiples contribuciones de una plataforma de e-learning " MoodleCloud " en un enfoque educativo híbrido enriquecería la instrucción en el aula y desarrollaría mejor las habilidades de comprensión lectora de estos estudiantes. Para verificar nuestras hipótesis de investigación, realizamos un estudio de campo de tres fases a lo largo de un semestre de estudio con estudiantes argelinos matriculados en el primer año de su licenciatura en el departamento francés. 
Palabras clave: dispositivo híbrido de formación, comprensión lectora, francés como lengua extranjera, pedagogía universitaria, contexto universitario argelino

\section{Introduction}

La réforme du système éducatif algérien promulguée en 2002 a mis l'accent sur la nécessité de former des lecteurs compétents en langue L2, et donc de développer l'apprentissage/enseignement de la compétence à lire et à comprendre les différents types de texte dans tous les niveaux d'enseignement, à l'école fondamentale, au collège, au lycée et même à l'université. Pourtant, de nombreux travaux de recherche telles que ceux de Ammouden \& Ammouden (2010), Kherra (2011), Ouhaibia (2016) et Sebane (2011), réalisés conformément aux niveaux décrits par le Cadre Européen Commun de Référence pour les Langues (CECRL) ont révélé que plus de $90 \%$ des étudiants algériens rejoignent les bancs de l'université en n'ayant qu'un niveau élémentaire en français (A1). D'autres constats établis à partir des travaux de recherche traitant les problèmes de l'apprentissage de la compréhension et production écrite (Boudechiche, 2008; Kherbache, 2008; Ouhaibia, 2010; Sebane, 2008) ont également dévoilé que beaucoup d'obstacles empêchaient les étudiants de développer suffisamment de compétences en littératie. Ces difficultés que les apprenants peinent souvent à surmonter sont particulièrement décisives pour leur réussite scolaire et leur insertion professionnelle.

C'est d'ailleurs dans cette perspective et avec l'avènement des TIC dans les milieux de formation que des chercheurs appartenant aux pays du Nord (Karsenti, 2004; Legros, 2006; Depover, Karsenti \& Komis, 2007; Assude \& Loisy, 2008; Lebrun, 2011) ont tenté d'expérimenter ces outils technologiques dans plusieurs situations d'enseignement/apprentissage des langues afin d'identifier les divers apports qu'ils sont en mesure d'offrir.

Nous souhaitons, à la lumière de ces travaux et dans le but d'apporter une solution pédagogique efficace aux difficultés rencontrées lors de l'apprentissage du module de "Linguistique ", examiner dans quelle mesure l'exploitation des multiples potentialités des dispositifs de formation hybrides pourraient aider à la compréhension en lecture des matières de spécialité.

Dans ce qui suit, nous présenterons en premier lieu un petit aperçu du contexte et des principes théoriques sur lesquels se base notre présente recherche; en second lieu nous exposerons, sans trop apporter de détails, la méthodologie qui sous-tend notre étude; finalement nous focaliserons notre réflexion sur l'expérimentation d'un nouveau dispositif de suivi et d'accompagnement mis en place pour faciliter les apprentissages des étudiants ainsi que sur l'analyse de ses effets.

\section{Contexte et problématique}

Cette contribution présente, à la lumière des travaux les plus récents, les principaux résultats d'une étude faite auprès d'étudiants algériens inscrits en première année Licence et qui repose sur la mise au point et l'expérimentation d'aides à la compréhension d'un texte explicatif en langue L2 (Français) exposant un module jugé comme complexe dispensé dans le département de Français : la linguistique. Les travaux de recherche qui se sont intéressés à la compréhension des textes explicatifs s'appuient sur les nombreuses recherches qui ont montré que "l'acte de comprendre » est une représentation cohérente de texte que le lecteur construit à partir des informations véhiculées dans le texte en y ajoutant ses propres connaissances du sujet emmagasinées dans sa mémoire qu'il développe à partir de processus inférentiels qui servent à 
expliquer et à mettre en évidence les rapports de cohérence existants entre les énoncés. Ces deux derniers processus reposent à leur tour sur deux autres composants du système cognitif qui sont la mémoire de travail (MDT) et la métacognition. Le traitement des anaphores et des inférences s'appuie sur l'aptitude du lecteur à garder actives les informations lues antérieurement (Cain, Oakhill, \& Bryant, 2004).

Néanmoins pour être capable de comprendre, il est également nécessaire que le lecteur comprenne et réalise qu'un traitement spécial est capital de sa part pour activer ce genre de processus. En effet, ce sont les processus métacognitifs (inférence, flexibilité, régulation, contrôle et adaptation) qui favorisent la bonne compréhension (Cain, Oakhill \& Bryant, 2004).

C'est pour cette raison que ces auteurs (2000) mettent l'accent sur l'importance de travailler la compréhension, pas seulement au primaire et au secondaire, mais aussi et surtout au supérieur. Ils défendent l'idée que la seule façon pour nous de soulever notre niveau de compréhension des apprenants est de perpétuer l'entrainement de cette compétence.

De ce postulat qui définit l'activité de lecture comme un apprentissage complexe et multidimensionnel en ce qui concerne les processus cognitifs (Bianco, 2015; Carliste \& Rice, 2002; Clarke, Truelove, Hulmes \& Snowling, 2014; Irwin, 2007), la lecture de textes de disciplines complexes et peu connues des étudiants ne peut être sans difficultés. Ceci est encore plus vrai lorsqu'il s'agit de textes produits en L2 ou LE. Le répertoire lexical de ces étudiants, généralement insuffisant, entre en conflit avec les données véhiculées directement ou indirectement dans le texte, ce qui mène ces étudiants à de grandes difficultés pour développer les inférences nécessaires à la construction de cohérence globale de la représentation du contenu sémantique du texte (Best, Row, Ozuru \& MacNamara, 2005). Ces obstacles poussent les étudiants à générer des représentations incomplètes, partiellement cohérentes, voire incohérentes la plupart du temps (Legros \& Baudet, 1996) et à activer des stratégies inutiles (Marin, Crinon, Legros \& Avel, 2007).

A l'encontre de ces difficultés, des projets de recherche ont été élaborés pour améliorer la compréhension et l'apprentissage/enseignement de la compréhension en lecture (Yee \& Mclntyre, 2013), notamment celles traitant les apports des TICE sur le traitement cognitif des textes (Baccino, 2004; Legros \& Crinon, 2002; Legros, Pudelko, Crinon \& Tricot, 2000; Mangen, Walgermo \& Brønnick, 2013; Tierney, 2009; Wright, Fugett \& Caputa, 2013), qui ont permis la création de plusieurs aides numériques multi et hypermédias (Closed Hypertext Systems) et des systèmes de co-compréhension à distance (Open-ended Internet Environments), qui ont fini par la suite par être ajustés pour une meilleure adaptation et soumis à des tests pour vérification (Al-Seghayer, 2005; Coiro, 2003; Legros, Hoareau, Boudechiche, Makhlouf \& Gabsi, 2007; McNamara \& Shapiro, 2005; Snow, 2010; Vidal-Abarca et al., 2002).

Un logiciel interactif et adaptatif nommé «iSTART » a été développé par McNamara, Levinstein \& Boonthum (2004) pour d'un côté, améliorer le développement des stratégies de lecture actives en s'ajustant au niveau de compétences des apprenants et à leur connaissance, et d'un autre coté pour aider à construire une meilleure représentation du contenu des textes, en planifiant des modules d'aide à l'apprentissage des stratégie telles que : la prédiction de la suite du texte, la génération d'inférences, l'utilisation de connaissances préalables et le contrôle de la compréhension. O'Reilly, Sinclair \& McNamara (2004) ont par la suite testé l'impact d'iSTART sur la compréhension d'un texte scientifique soumis à 38 élèves, à l'aide de questions se rapportant soit aux informations véhiculées dans le texte soit aux connaissances sous-entendues relevant des inférences. Il s'est révélé que les élèves ayant suivis un entrainement à l'aide du logiciel ont de meilleures performances en compréhension de lecture de textes scientifiques que les élèves non entrainés avec iSTART. Ceci confirme bien l'hypothèse qui sous-tend que le logiciel iSTART améliore la compréhension en lecture de textes scientifiques. 
D'autres travaux se sont aussi intéressés à l'importance du questionnement sur le développement de connaissances par l'activation de stratégies efficaces de compréhension pendant les activités de compréhension en lecture, précisément la lecture de textes produits en L2. Mentionnons l'étude de Kazuhiro (2008), qui servait à mesurer l'effet de deux genres de questions (des questions sur le contenu du texte et des questions inférentielles) sur la compréhension de textes produits en L2 en questionnant deux groupes d'étudiants japonais séparés en trois niveaux (faible, intermédiaire et bon) qui avaient chacun pour consigne de traiter un seul genre de question. Dans cette étude, Kazuhiro a démontré que les questions se rapportant au contenu du texte amélioraient surtout la compréhension des participants ayant un bon niveau, tandis que les questions inférentielles impactaient la compréhension de tous les participants sans exception.

Sans oublier aussi qu'avec le développement des outils multimédias, d'autres percées technologiques ont été réalisées, telles que : la création de systèmes de questionnement numérique qui permettent d'ajuster l'entrainement au niveau de compétence des apprenants sur les domaines de connaissances présentés par le texte (closed hypertext systems) (Dalton \& Proctor, 2007; Kangsepp, 2011), et également le développement de bases de données ainsi que l'apprentissage en ligne qui ont largement montré leur efficacité notamment à travers différents travaux de recherches (Fan, 2012; Ghorbani \& Nezamoshari'e, 2012; Momtaz \& Garner, 2010; Pan \& Wu, 2013; Zuo, 2011) dans lesquels ils ont su prouver que les échanges entre pairs favorisent efficacement la compréhension des textes et la construction de connaissances.

De plus, depuis une dizaine d'années l'exploitation des plateformes d'enseignement s'est propagée dans les universités européennes, leur faisant ainsi subir une transformation des dispositifs traditionnels vers les nouveaux dispositifs hybrides qui reposent sur l'articulation entre le présentiel, la distance et les environnements techno-pédagogiques (Charlier, Deschryver et Peraya, 2006). Cependant, peu d'études révèlent un impact positif de ces transformations sur la qualité des processus d'enseignement/d'apprentissage. Ce n'est que récemment que la réflexion s'est intéressée sur l'importance d'accorder de l'intérêt au dispositif pédagogique encadrant les outils et à l'attention pédagogique devenue plus cruciale en ce qui concerne les dispositifs s'appuyant sur les technologies. Selon Clark (1994) et Tardif (1996), une pédagogie rigoureuse est une condition indispensable pour que les TIC (technologies de l'information et de la communication) tiennent parole et ouvrent l'accès à une formation de qualité. C'est pour cette raison que l'alignement pédagogique des objectifs, des méthodes et des évaluations (Biggs, 1996) que nous complétons par l'utilisation des outils technologiques (Lebrun, 2007) représentent un élément indispensable d'un effet positif des TIC : «Our review provides convincing evidence that information technology can enhance learning when the pedagogy is sound, and where there is a good match of technology, techniques and objectives " (Kadiyala \& Crynes, 2000). Cela nous parait nécessaire pour palier la part supposée de ces impacts que Morgan (2003) définissait ainsi :

There is little empirical evidence that course management systems actually improve pedagogy. Study findings suggest, however, that using a CMS does invite faculty to rethink their course instruction and instructional environment, resulting in a sort of accidental pedagogy.

Au travers de notre travail de recherche, nous souhaitons expérimenter de façon empirique l'effet de ces technologies sur l'optimisation de la compréhension en lecture des étudiants algériens et soutenir davantage l'idée que les valeurs ajoutées qu'offrent les technologies à l'apprentissage exigent des dispositifs adéquats conçus sur des pédagogies actives, incitatives et interactives.

Dans une perspective socio-constructiviste de l'apprentissage, nous voulons montrer en quoi un dispositif d'apprentissage hybride soutenu par la plateforme « MoodleCloud » pourrait améliorer la compréhension en lecture d'étudiants algériens inscrits en première année Licence. 
En effet, nous pensons qu'exploiter soigneusement les potentialités d'une plateforme d'apprentissage en ligne dans le cadre de la mise en place d'un dispositif hybride de formation peut également permettre le développement d'une activité réflexive plus importante. En effet, vu que la plateforme "MoodleCloud » intègre des ressources et des activités d'apprentissage tels que : les glossaires, forum, test, feedBack, wiki, chat, etc., qui permettent le dialogue (communication entre pairs), l'évaluation (voire l'autoévaluation) et la collaboration, elle permet donc une confrontation du traitement différencié de l'information. Traitement qui selon Van Dijk \& Kintsch (1983), s'effectue en trois temps. Le premier temps est dit " code de surface » : il correspond aux caractéristiques lexicales et sémantiques du texte. Le second temps est appelé « base de texte " : c'est un traitement qui s'opère sur l'ensemble des propositions sémantiques traduisant le contenu explicite du texte à étudier. Quant au troisième, nommé « Modèle de situation », c'est un processus qui se déploie sur l'ensemble des connaissances évoquées par le texte à étudier et qui met en relation le contenu du texte à étudier avec les connaissances du lecteur. C'est la confrontation de ce triple traitement que réalisent les co-compreneurs lors de l'activité de compréhension qui pourrait mener à une productivité plus rigoureuse et plus efficace aidant ainsi les étudiants à construire des compétences spécifiques à la compréhension de textes produits en L2 en contexte plurilingue et diglossique. Si cette nouvelle méthode d'enseignement/apprentissage se révèle efficace, son intégration et exploitation dans l'enseignement supérieur développerait considérablement les compétences littératiques des étudiants et par conséquent élever leurs niveaux.

Nous supposons que l'exploitation des multiples fonctionnalités d'une plateforme d'apprentissage en ligne dans le cadre d'une formation hybride enrichit l'enseignement dispensé en classe et développe au mieux la compétence de compréhension en lecture des étudiants.

Cette principale hypothèse sous-tend deux sous-hypothèses :

1) L'effet d'un cours préparé et didactisé au moyen de la chaine éditoriale « Scénari-Opale » sur la motivation des étudiants et le développement de leurs compétences en compréhension écrite.

2) L'effet de la mise en place d'activités en ligne destinées à la communication, l'évaluation formative et la construction collaborative de contenus sur l'amélioration de la compréhension en lecture de textes explicatifs proposés aux étudiants de première année. (Khelfi, Zarrouk Ben Abid et Kadi Ksouri, 2018)

\section{Méthodologie}

Dans le but d'assurer l'efficacité, l'attractivité et la fiabilité du dispositif, nous avons dans un premier temps effectué une analyse du contexte d'investigation, des contenus pédagogiques proposés et des objectifs attendus ainsi que de la littérature qui s'est intéressée à l'approche par tâche et aux formations hybrides. Puis, dans un second temps nous avons planifié des rencontres avec l'enseignant et les étudiants pour d'un côté, dissiper les craintes et hésitations ressenties à l'encontre de l'utilisation des technologies dans le cadre de leur formation et de l'autre, fournir quelques informations d'ordre théorique et technique en rapport avec la mise en place du dispositif, nécessaires pour construire une équipe totalement engagée dans la réalisation du projet.

\section{Participants}

Durant le deuxième Semestre de l'année universitaire 2017/2018, nous avons procédé à une enquête quasi-expérimentale auprès de 26 inscrits en première année Licence au département de Français à l'université de Badji Mokhtar Annaba. 
Les sujets de notre étude sont âgés entre 19 et 25 ans. Chaque groupe est constitué de 8 filles et de 5 garçons, qui ont été répartis en 2 groupes :

- Un groupe quasi-expérimental ayant participé à une formation hybride articulant des séances de formation en présentiel et hors classe par le biais d'une plateforme pédagogique.

- Un groupe témoin ayant suivi un enseignement traditionnel dispensé uniquement en salle de cours en compagnie de l'enseignant.

\section{JUSTIFICATION DU CHOIX DES PARTICIPANTS}

Pour la rentrée universitaire 2017/2018, 277 bacheliers partagés en 7 groupes ont rejoint les bancs des salles de cours du département de Français.

Sur les sept groupes de première année, nous n'avons gardé que deux groupes pour la $2^{\mathrm{e}}$ phase de notre enquête. Ce choix n'était pas assigné au hasard, il est justifié par une pré-enquête exploratoire menée durant trois semaines auprès de toute la promotion, qui a permis de recueillir 170 réponses sur un total comptant 277 apprenants, ceci à cause du taux élevé d'absentéisme répété auquel nous avons dû faire face en administrant notre questionnaire.

Au travers de cette pré-enquête, plusieurs conclusions ont été déduites. Trois d'entre elles ont été capitales pour la suite de notre enquête :

- La première conclusion concerne l'usage et le niveau de connaissances des Technologies de l'information et communication (TIC) par les questionnés.

\section{Quel(s) média(s) possédez-vous ?}

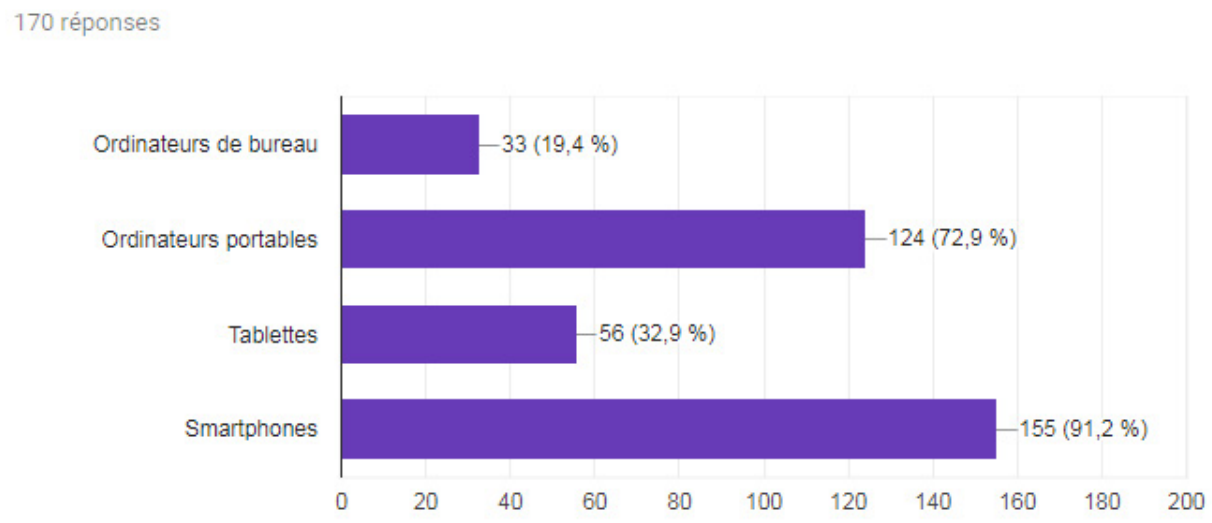

Figure 1. Outils technologiques utilisés par les étudiants 
Selon quelle fréquence?

169 réponses

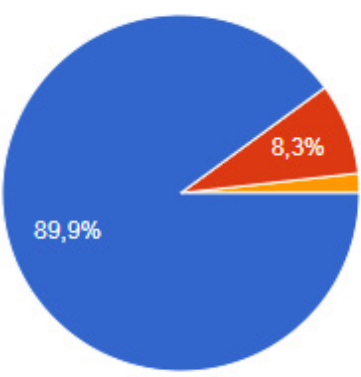

Plus d'une fois par jour

Plus d'une fois par semaine

Plus d'une fois par mois

Figure 2. Fréquence d'utilisation des outils technologiques par les étudiants

\section{Quelle est votre fréquence de connexion à Internet ?}

170 réponses

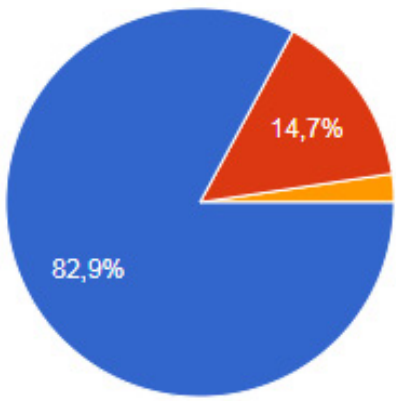

Plus d'une fois par jour

Plus d'une fois par semaine

Plus d'une fois par mois

Figure 3. Fréquence d'utilisation d'Internet par les étudiants 
Comment qualifierez-vous votre niveau d'utilisation de l'ordinateur?

170 réponses

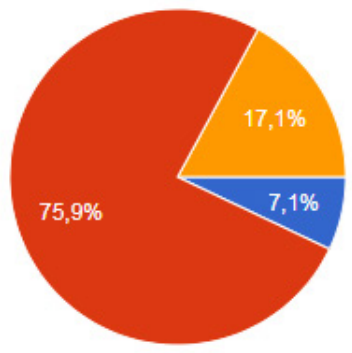

Figure 4. Niveau d'utilisation de l'ordinateur par les étudiants

\section{Vous arrive-t-il de vous connecter sur les réseaux sociaux ?}

\section{8 réponses}

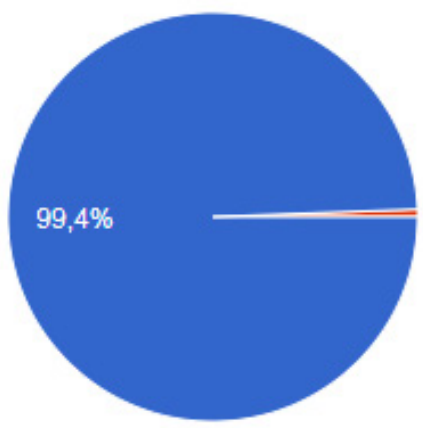

Oui

non

Figure 5. Taux d'étudiants qui utilisent les réseaux sociaux

Les figures 1 à 5 nous ont permis de déduire deux choses : la première est que la majorité des enquêtés disposent d'Internet et d'outils technologiques et les utilisent plus d'une fois par jour aussi bien dans le cadre de leur étude que de leurs loisirs; la deuxième concerne le degré de connaissance des questionnés, à savoir que la majorité des questionnés ont un niveau Moyen en informatique et savent tous utiliser les réseaux sociaux. Ce niveau de connaissances est largement suffisant pour notre étude. 
- La deuxième conclusion porte sur la compétence et le module qui posent le plus problèmes aux étudiants.

Parmi les modules de 1 ère année, quel est celui que vous avez le plus de mal à comprendre en lisant le contenu de ses cours ?

170 réponses

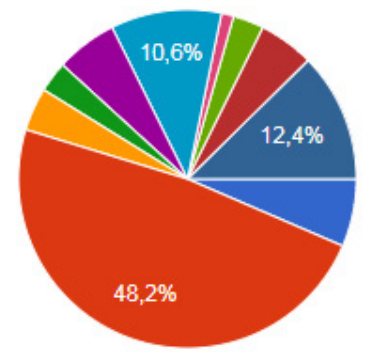

Méthodologie du travail universitair.

Linguistique

Grammaire

Technique d'expression orale (TEO)

Technique d'expression écrite (TEE)

Culture, civilisation

Anglais

Sciences sociales

A $1 / 2 \nabla$

Figure 6. Module que les étudiants ont le plus de mal à acquérir

Parmi ces quatre compétences, quelle est celle qui, pour vous, est la plus difficile?

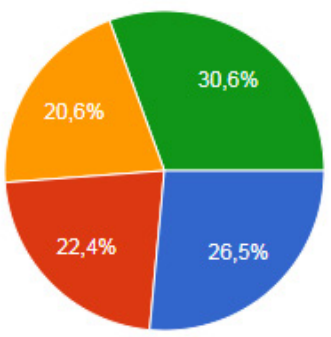

Expression orale

Expression écrite

Compréhension orale

Compréhension écrite

Figure 7. Compétence que les étudiants peinent à acquérir

Au travers des figures 6 et 7 , nous avons pu en tirer que dans le stade actuel de l'apprentissage de la population questionnée, $48,2 \%$ d'entre eux ont le plus de mal à comprendre le contenu sémantique des leçons dispensées dans le module de "Linguistique ».

- La troisième conclusion repose sur le nombre d'étudiants souhaitant participer à la deuxième phase de notre étude : la quasi-expérimentation. 


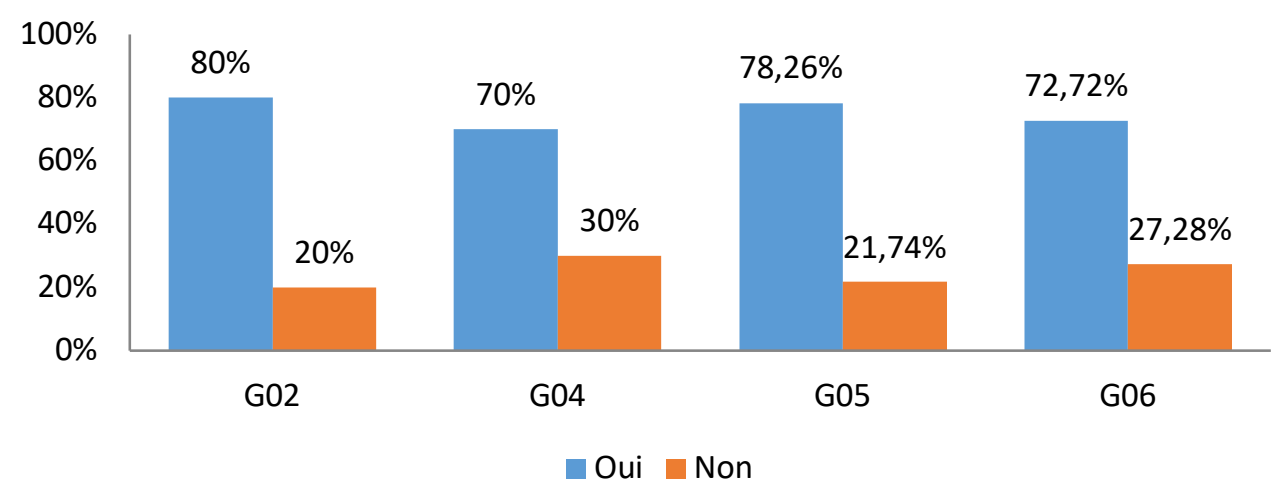

Figure 8. Taux d'étudiants ayant accepté de participer au projet

Comme, dans le cadre de notre recherche il est nécessaire que nos sujets suivent une seule et même approche d'enseignement/apprentissage, nous avons dû dans un premier temps retenir 4 groupes d'étudiants (figure 8).

Puis, une autre sélection a été opérée, basée sur le nombre de questionnés ayant répondus favorablement pour participer durant environ un mois à une formation dispensée en classe et complétée, voire renforcée par un suivi à distance à l'aide d'une plateforme d'apprentissage (voir la figure 8). Les étudiants qui ont été retenus comme groupe " quasi-expérimental » sont ceux qui ont le plus répondu " Oui » et ceux qui ont le moins répondu « Oui » ont été pris comme groupe « témoin ».

\section{DESCRIPTION DE LA PROCÉDURE EXPÉRIMENTALE}

En classe, les participants des deux groupes ont bénéficié de la même approche d'enseignement/apprentissage d'un même cours. Ils ont reçu parallèlement un même enseignement d'une durée de 3 heures pendant deux semaines, à raison d'une heure et demie par semaine.

Néanmoins, le groupe expérimental a, en plus des enseignements et activités prévus en présentiel, bénéficié d'un suivi à distance via une plateforme d'apprentissage " MoodleCloud " d'une durée de 12 heures pendant deux semaines, à raison de 6 heures par semaine partagées en deux séances chacune de 3 heures, lors desquelles ils ont profité d'un enseignement combinant plusieurs types d'apprentissage : un apprentissage à partir d'exemples en situation de résolution de problèmes, un apprentissage collaboratif et un apprentissage par évaluation formative continue (Farza, 2015).

En effet, les participants à la formation hybride ont, en plus des séances programmées en classe, consulté à distance leur cours préparé en amont sur la chaine éditoriale "Scénari Opale » et mis en ligne sur la plateforme " MoodleCloud », accompagné d'activités d'apprentissage telles que : tests, chat, forum, wiki, glossaire...etc. Nous avons volontairement mis ces activités à leur disposition pour d'un côté, réguler leur compréhension en les évaluant régulièrement, et de l'autre favoriser la collaboration et la coopération entre eux, ce qui peut se révéler être une tâche constructive et productive.

A la fin de notre enquête, nous avons planifié un test de connaissances pour les deux groupes en même temps et pour une même durée afin de détecter le degré de leur acquis d'apprentissage et de mesurer leur compréhension en lecture. 
Cependant avant d'entamer notre enquête, nous avons élaboré une formation, que nous avons par la suite présentée aux étudiants pendant trois heures, afin de leur expliquer un nouveau mode de fonctionnement en rupture avec leurs habitudes, relativement à une approche d'enseignement hybride soutenue par une plateforme d'apprentissage; tout cela dans le but d'assurer l'efficacité et la fiabilité du protocole qui soustend notre étude.

\section{Instruments de collecte et analyse des données}

Pour le recueil de données, nous avons utilisé trois outils : un questionnaire initial à valeur exploratoire, un test de connaissances sous forme d'une évaluation sommative et un questionnaire d'appréciation de la formation hybride administré aux étudiants.

\section{QUESTIONNAIRE INITIAL}

Notre questionnaire initial comprenait 30 questions à choix unique et multiple regroupées en 4 rubriques : une rubrique "Usages », une rubrique "Connaissances », une rubrique " Méthodes et représentations » et une rubrique " Informations générale ». Nous l'avons administré à titre exploratoire aux 7 groupes de première année Français pour deux raisons :

1) pour mesurer leur niveau d'utilisation et connaissances des TICE;

2) pour découvrir la compétence et la matière qu'ils avaient le plus de mal à assimiler et acquérir.

Les résultats obtenus à partir du questionnaire étaient déterminants pour la suite de notre enquête. Ils nous ont permis, d'une part, d'affirmer que les groupes étaient bien comparables et, d'autre part, d'opérer un ensemble de choix nécessaires concernant les étudiants qui participeront en tant que groupe « quasiexpérimental » et « témoin » à la deuxième phase de notre enquête.

\section{TEST DE CONNAISSANCES SOUS FORME D'UNE ÉVALUATION SOMMATIVE}

En collaboration avec l'enseignant chargé du module, nous avons programmé une évaluation sommative aux deux groupes pour une même journée et une même durée. Le sujet de l'évaluation comprenait 4 questions auxquelles les étudiants devaient répondre. Nous avons fait en sorte que chaque question formulée interroge une partie du cours présenté en classe et à distance pour pouvoir mesurer et évaluer convenablement et avec précision l'ensemble des connaissances acquises par les étudiants.

\section{QUESTIONNAIRE D’APPRÉCIATION DE LA FORMATION HYBRIDE}

A la fin de l'évaluation sommative, les étudiants du groupe quasi-expérimental ont été invités à renseigner anonymement un questionnaire comprenant 15 questions fermées que nous avons formulées en nous référant sur la méthode de Tricot et al. (2003) afin de connaitre leurs appréciations ou dépréciations face à cette nouvelle technique d'enseignement/apprentissage hybride.

\section{Méthode d'analyse des données}

Dans une approche d'évaluation de la qualité des réponses, fournies par les étudiants des deux groupes lors de l'évaluation sommative, qui constituent le corpus principal de notre étude, il est nécessaire de définir avec précision les critères et outils d'analyse et d'appréciation et de confirmer avec exactitude qu'ils s'accommodent bel et bien aux objectifs et paramètres de notre enquête. 
Dans notre recherche, il est question de mettre en évidence des indicateurs pertinents qui nous permettent d'apprécier la qualité des réponses émises par les étudiants du groupe ayant participé à une formation alternant le présentiel et la distance et ceux du groupe ayant suivi le même cours uniquement en classe. Cette démarche vise à identifier les différences possibles entre les réponses produites par les deux groupes, aussi bien au niveau linguistique qu'au niveau référentiel, et de détecter les potentielles valeurs ajoutées qu'une approche de formation hybride peut procurer.

De ce fait, pour examiner les copies des étudiants, nous nous sommes appuyées sur une série de grilles d'analyse croisant des critères qui, à nos yeux, sont à même de mettre en relief les différences pouvant exister dans les réponses proposées par les étudiants :

1) Grille relative au corrigé-type de l'évaluation sommative appliquée selon la méthode des juges.

2) Grille relative à l'analyse propositionnelle proposée par Van Dijk \& Kintsch (1983) et Kintsch (1998) et à la pertinence du contenu sémantique soulevée par Sperber \& Wilson (1989).

3) Grille relative aux erreurs orthographiques et grammaticales.

4) Grille relative au mode de lecture du texte expérimental.

Une telle démarche d'analyse permet de constater qu'il existe une cohérence et une pertinence au niveau des réponses formulées; la démarche permet aussi d'identifier le degré d'intégration des nouvelles connaissances par les étudiants, en fragmentant chaque énoncé en proposition et en émettant un jugement de vérité (Tiberghien, 1997). Bien qu'elle ne soit pas sans risque, cette démarche est la plus appropriée, puisque les jugements de vérité proposés peuvent contenir une part de subjectivité. C'est d'ailleurs pour cette raison que nous avons fait appel à la méthode des juges, en sollicitant l'aide de trois enseignants de linguistique pour corriger des copies anonymes des étudiants ayant participé activement à l'enquête et pour leur attribuer des notes.

Quant à l'analyse des réponses aux questionnaires initial et final, il est à préciser que seules des fréquences ont été calculées. 


\section{Analyse des résultats}

\section{Analyse des résultats de l'évaluation sommative}

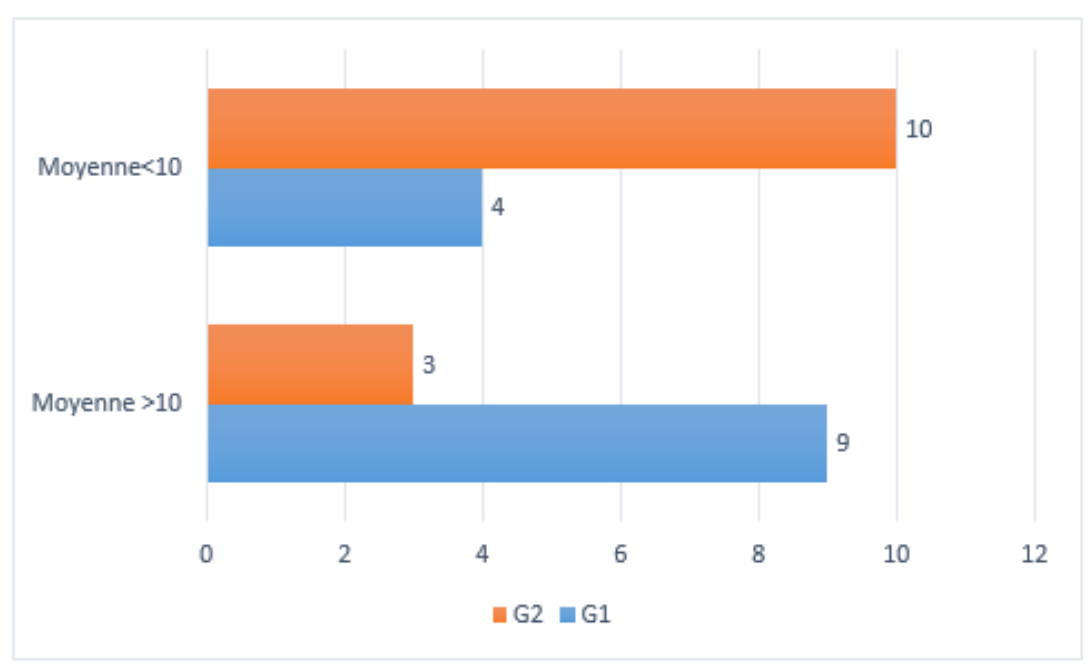

Figure 9. Moyennes générales que les étudiants ont obtenues à l'évaluation sommative

En complément de la figure 9, mentionnons que la moyenne générale des notes attribuées aux étudiants du groupe expérimental (G1) par les trois juges est de 7.10, nettement au-dessus de la moyenne, contrairement à la moyenne générale du groupe témoin qui est de 3.90/10 (G2). En partant de ces données, nous pouvons déclarer qu'un enseignement traditionnel complété par un suivi à distance a un effet positif sur les étudiants et permet d'accroitre considérablement leurs performances de compréhension en lecture. 


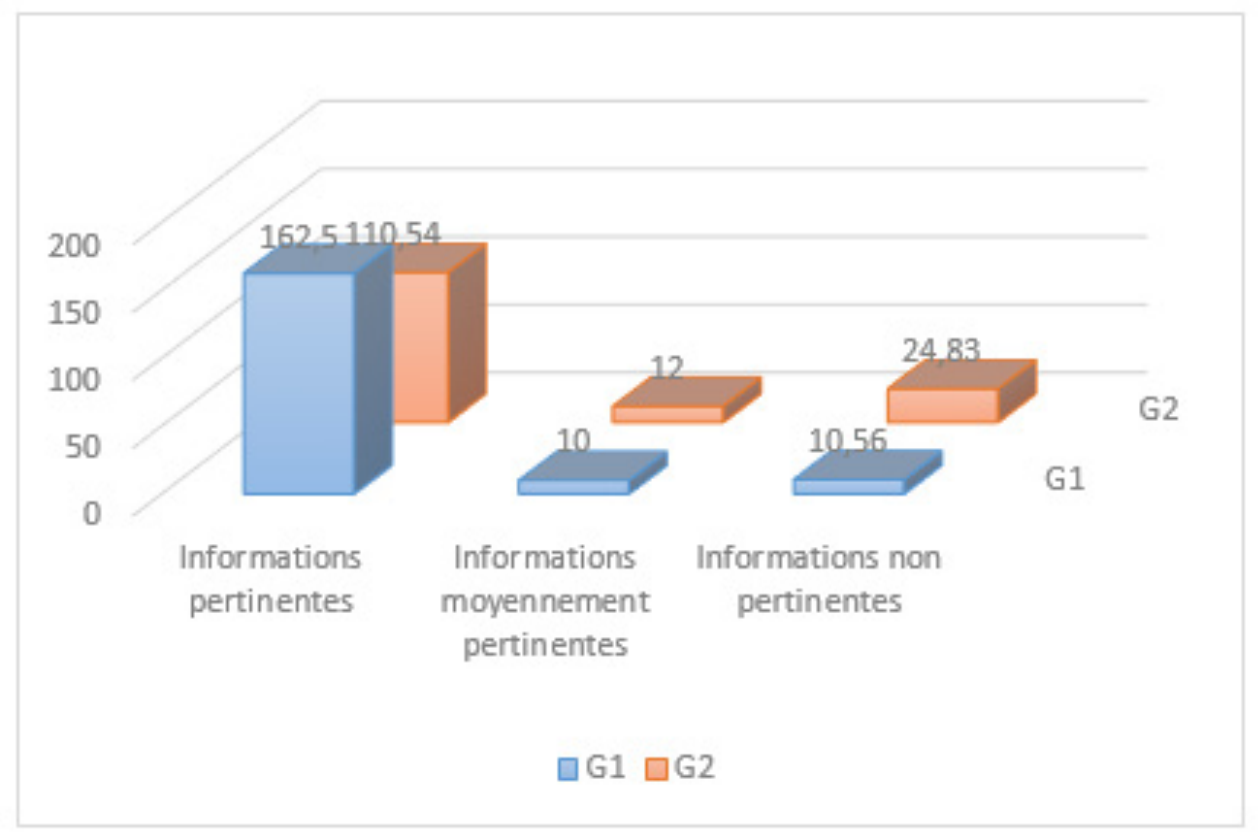

Figure 10. Taux d'informations activées par les étudiants lors de l'évaluation sommative

Comme l'indique la figure 10, l'analyse du niveau sémantique a révélé que les étudiants du groupe expérimental ont produit plus d'informations pertinentes (162,5exp vs 110,54tém), moins d'informations moyennement pertinentes (10exp vs 12tém) et beaucoup moins d'informations non pertinentes que le groupe témoin $(10,56 \mathrm{exp}$ vs 24,83 tém). Nous pouvons finalement noter que le mode de lecture du texte expérimental (sur écran vs sur papier) impactent vraisemblablement l'activation des informations. En effet, les participants du groupe expérimental G1 qui ont lu le cours sur écran ont activé plus d'informations que les participants du groupe témoin qui ont lu le même cours sur polycopié (183,03exp vs 147,37tém).

A partir de cette analyse, nous avons pu tirer deux conclusions :

1) Lire et consulter un cours sur écran, par le biais d'une plateforme d'apprentissage créée et développée dans le but d'apporter une solution pédagogique, aide à améliorer la compréhension en lecture des étudiants.

2) Insérer des bulles d'informations dans le contenu pédagogique, ajouter des liens hypertextes permettant de consulter d'autres sources d'informations vérifiés et contrôlés préalablement par l'enseignant et utiliser des dictionnaires de spécialité, aident à mieux comprendre les mots difficiles et à intégrer des nouvelles connaissances. 


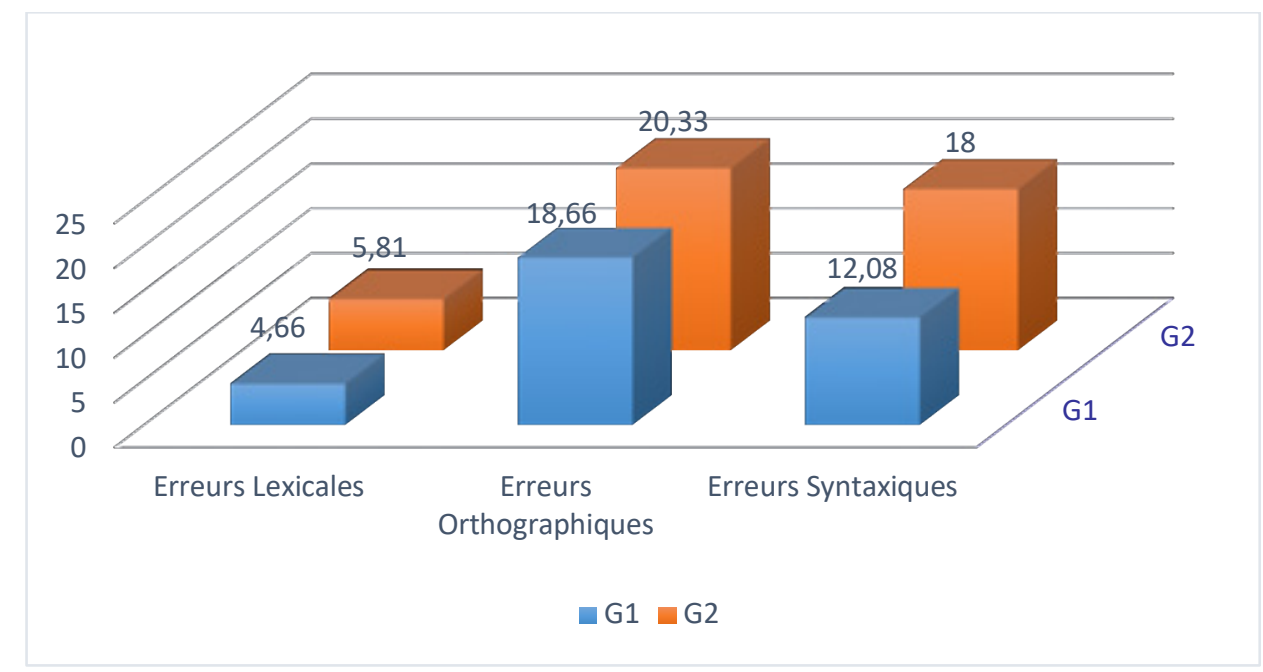

Figure 11. Taux d'erreurs commises par les étudiants lors de l'évaluation sommative

Tel que représenté dans la figure 11, l'analyse du niveau de surface a nettement démontré que les erreurs linguistiques commises par le groupe témoin (G2) sont beaucoup plus nombreuses et importantes que ceux du groupe expérimental (G1); nous pouvons en déduire qu'une lecture sur écran aide à mieux retenir les concepts clés évoqués dans le cours.

En partant de ces deux analyses, nous pouvons affirmer sans crainte que finalement un accompagnement en dehors de la classe en utilisant une plateforme d'apprentissage est bénéfique et améliore considérablement le niveau de compréhension en lecture des étudiants.

\section{Analyse des résultats du questionnaire d'évaluation de la formation}

\section{Les activités d'apprentissage proposées par votre enseignant pour contrôler votre compréhension (chat, forum, tests...etc) vous ont parues}

\section{3 réponses}

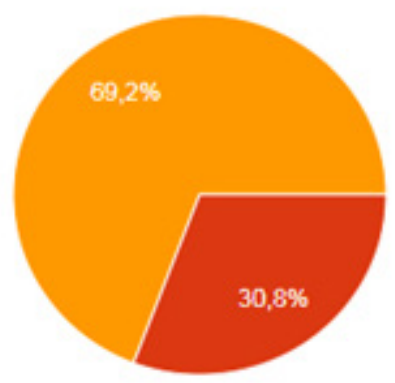

Figure 12. Utilité des activités d'apprentissage disponibles sur les plateformes d'apprentissage 


\section{S'il vous est demandé de formuler à vos enseignants quelques}

propositions en vue d'améliorer davantage vos formations et vos apprentissages, est ce que l'application de cette nouvelle méthode d'Enseignement/Apprentissage hybride en ferait-elle partie?

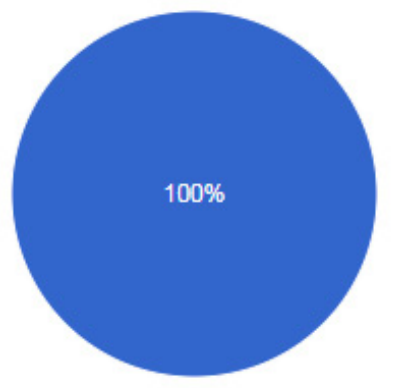

Figure 13. Taux d'étudiants souhaitant renouveler l'expérience

D'après les données représentées dans les figures 11, 12 et 13, nous notons clairement l'existence d'une certaine satisfaction de la part des étudiants ayant participé à la formation hybride; nous notons également un important enthousiasme puisque la totalité des participants, qui au début étaient totalement contre l'idée de prolonger les heures de cours en dehors de la classe, souhaitent renouveler l'expérience et convaincre le reste de leurs enseignants de l'adopter.

\section{Recommandations}

A l'issue de notre enquête, il a été possible de proposer une série de recommandations permettant une mobilisation plus efficace de la formation hybride :

\section{Réduire les obstacles techniques}

Afin de remédier aux éventuelles difficultés émanant de l'utilisation des TIC pour la création et la diffusion des cours, il est souhaitable de recourir à des logiciels et plateformes spécialisés créés en guise de solution pour ce genre de handicap, tels que : la chaine éditoriale Scénari Opale, la plateforme Moodle, etc.

Aussi, afin d'éviter les embuches liées à l'accès Internet ou encore aux outils technologiques pour consulter les cours en ligne, il faut noter que la majorité des universités possèdent de nos jours des environnements informatisés dotés d'une connexion Internet haut débit. Certains établissements de formation se portent garant et prêtent totalement des ordinateurs portables à leurs étudiants, tout cela pour s'assurer qu'ils ont bien pu accéder et consulter leurs leçons. En cas de manque de moyens technologiques suffisants, il serait plus simple de se rapprocher de son institution de formation et de solliciter leurs interventions.

De même, pour pallier les insuffisances liées au manque de connaissances et compétences technologiques, il serait judicieux de la part des institutions et enseignants de prévoir et proposer des séances de formation à ceux qui sont dans le besoin. 


\section{Veiller à proposer aux apprenants des ressources de bonne qualité, cohérente et en nombre limité}

Les contenus de cours constituant l'élément central de toute formation, il est important de leur accorder une attention particulière. De plus, étant donné le caractère distantiel de la formation, les concepteurs de contenus et de supports doivent tout mettre en œuvre pour que l'apprenant soit captivé par le cours. L'une des astuces pour satisfaire les apprenants des formations en mode e-learning ou mixte (présentielle et mode e-learning) est de créer des contenus de cours animés, interactifs et agréables à l'apprentissage. C'est pourquoi à notre sens il est nécessaire de faire appel à l'approche Chaine éditoriale précisément la chaine éditoriale "Scénari ». Cette chaine, apparue dans le but de pallier les inconvénients occasionnés par l'approche bureautique et LaTex, permet à son auteur de disposer d'un seul et même outil pour produire un contenu et automatiser sa mise en forme. En effet, comme c'est une solution "Open Source » s'inscrivant dans une approche WYSIWYM (What You See Is What You Mean), le point essentiel sur lequel elle veille est la rédaction du contenu et sa structuration. L'auteur qui l'adopte ne se focalise que sur le sens de l'information à véhiculer et se décharge ainsi des aspects liés à la mise en forme. Son utilisation permet de générer des fonds documentaires de type multimédia, autrement dit, un document combinant différents médias (textes, images fixes ou animés, sons, vidéo, graphiques, dessins) dans un même format numérique. Néanmoins, l'auteur procédant ainsi doit respecter un modèle documentaire qui l'aide à structurer son contenu tout en le déchargeant de la mise en forme du produit final. Parmi les modèles documentaires fondés sur Scénari, Opale est le modèle spécifique destiné à la création de contenus pédagogiques. II représente un ensemble d'outils logiciels et méthodologiques qui permettent la gestion et la publication multi-support et multi-cible de contenus pédagogiques. II offre plusieurs avantages, dont :

- la priorité accordée au contenu et à sa structuration,

- la possibilité d'intégrer des ressources externes,

- la production automatisée multi-supports et multi-cibles qui permet de réutiliser les contenus et de produire différents assemblages des éléments du contenu pour différents profils d'apprenants.

- La possibilité et la facilité d'indexation des ressources.

De plus, son utilisation ne nécessite pas de faire appel aux services d'un développeur de logiciels ou d'avoir des connaissances en programmation d'information. $Y$ recourir dans le cadre d'une formation hybride est préférable si l'on souhaite bien sûr optimiser les apprentissages.

Pour ce qui est de la diffusion des contenus pédagogiques, exploiter les outils déjà existants tels que les plateformes d'apprentissage institutionnelles serait d'une aide considérable, surtout qu'ils ont été développés et acquis par les établissements de formation pour accompagner et faciliter aux enseignants la gestion de leurs cours en ligne. Ils pourraient bien les utiliser pour créer des contenus pédagogiques mais comme très peu d'outils sont intégrés à ces LMS, ils ne permettent que de générer des contenus propres au format de la plateforme et par conséquent, réduisent la possibilité de produire des cours multisupports, ce qui augmente considérablement la charge de travail de l'enseignant lorsqu'il souhaitera réutiliser ses cours dans d'autres contextes.

Cependant, l'exaltation des enseignants face aux nombreux outils technologiques existants peut engendrer une surcharge de travail non négligeable pour la réussite d'un cours. D'ailleurs, Sales (2013) met en garde contre la surabondance de matériel placé en ligne, surtout s'il porte sur un contenu facultatif (secondaire) au cours; cela ne fera que surcharger inutilement les étudiants puisqu'ils se sentiront obligés de tout consulter. Selon ce même auteur, il serait judicieux de mettre à la disposition des étudiants seulement les éléments qui leurs serviront et sans lesquels la réussite du cours ne peut être assurée. 
II faut également rester vigilant face à la démotivation des étudiants : ils risquent de contester cette nouvelle technique d'enseignement hybride en déclarant être trop surchargé, il faut constamment leur préciser que ce n'est qu'une impression et que bien au contraire, grâce à cette nouvelle façon de procéder, leur temps d'études sera allégé.

\section{Aider les enseignants dans le développement pédagogique}

II a été prouvé, et même remarqué par les enseignants, que leur charge de travail dans le cadre d'un enseignement hybride est beaucoup plus importante que lors d'un enseignement traditionnel. Pour éviter que cette surcharge ne se ressente davantage, des pistes de solutions concernant la création de contenus pédagogiques et l'évaluation des tests (devoirs) ont été proposées dans la littérature.

Bien que tout enseignant soit capable de concevoir seul son matériel didactique, deux stratégies diminueraient considérablement l'effort fourni par chacun dans l'accomplissement des différentes tâches : se réunir en groupe pour élaborer des activités d'apprentissage interactif et créer des contenus pédagogiques adaptés aux profils hétérogènes des étudiants. Encourager donc les enseignants à travailler en collaboration représente un contexte favorable pour l'implantation d'une formation hybride.

L'évaluation des travaux accomplis par les apprenants est une tâche que la plupart des enseignants souhaiteraient pouvoir déléguer à d'autres. Recourir à une méthode qui repose essentiellement sur l'exploitation d'une plateforme d'apprentissage faciliterait grandement la tâche aux tuteurs puisque grâce aux ressources qu'elles intègrent, les évaluations deviennent automatisées, ce qui devient un précieux gain de temps. Aussi, un tel dispositif hybride de formation permet l'évaluation par les pairs et l'autoévaluation; l'exploiter faciliterait encore plus le travail du tuteur qui, le plus souvent, se retrouve face à des classes surchargées d'apprenants. Les faire travailler en groupe pour mettre en commun leurs réponses serait rentable autant pour les tuteurs que pour les tutorés. Pour les apprenants, elle leurs permet de participer à une activité productive et constructive. Quant aux enseignants, elle leur offre la possibilité de corriger un seul devoir par équipe.

\section{Discussions et Conclusion}

Même si l'effet d'un dispositif de formation hybride sur la réussite scolaire n'a pas été explicitement prouvé, recourir à une telle méthode en situation pédagogique profiterait grandement aux deux groupes d'acteurs.

Du côté des étudiants, cette technique permet de satisfaire les besoins de certains apprenants se trouvant en difficultés et face à des situations handicapantes, en leur accordant la possibilité d'apprendre à leur rythme et selon les modalités qui leurs conviennent le mieux. Néanmoins, cette approche n'est pas sans difficultés. En plus des difficultés d'ordre technique qu'elle peut occasionner, certains étudiants témoignent à son encontre un important mécontentement relativement à deux aspects. D'une part, au changement de rôle que vit le pôle "enseignant", qui de l'unique détenteur du savoir devient un simple " accompagnateur ». D'autre part, à l'impression qu'ils ont sur la charge de travail qu'une telle approche implique: ils la perçoivent comme étant une tâche supplémentaire qui suppose de fournir un effort beaucoup plus important que celui fourni dans le cadre d'un enseignement magistral.

Pour ce qui est du point de vue des enseignants, une formation hybride procure aux étudiants la possibilité de bénéficier plus fréquemment de feedback, ce qui peut s'avérer être un atout majeur pour identifier ceux qui sont en difficulté. Cette technique peut aussi aider à rendre l'enseignement moins récurrent et plus varié. Par contre, la mise en place d'une telle approche implique une charge de travail additionnelle surtout en début d'implantation. 
Toute approche d'enseignement/apprentissage présente des inconvénients (des limites). C'est pourquoi, pour optimiser au mieux ce dispositif de formation, des pistes de recommandation portant sur des conseils d'ordre technique ont été proposées afin de limiter et contourner les obstacles pouvant résulter de ce type d'approche. Ces pistes de recommandation favorisent l'utilisation de ressources d'apprentissage performantes, adéquates et en bon nombre ainsi que l'accompagnement des enseignants dans le développement de leur formation. Le recueil de données, collectées à travers ce présent travail de recherche, soutient l'idée que l'utilisation d'une approche de formation hybride dans une classe de FLE pourrait améliorer la compréhension en lecture des cours de spécialité, puisqu'elle permet à l'étudiant de bénéficier d'un encadrement personnalisé, proposé par son enseignant, et cela même en dehors de la classe. Cependant, il est à noter que les contraintes liées à l'accès et l'utilisation des technologies de l'information et de la communication représentent un véritable frein pour le développement et l'adoption de cette approche, nouvellement connue par la plupart des enseignants et étudiants rencontrés dans le cadre de ce travail de recherche.

L'attention accordée aux dispositifs de formation hybride par les chercheurs des pays du Nord ainsi que les multiples recommandations et constats établis dans le cadre de cette recherche constituent selon nous une contribution pertinente au champ de la pédagogie de l'enseignement supérieur puisqu'une exploitation efficace d'une telle approche, accompagnée d'un soutien adéquat, peut aider à améliorer les environnements d'apprentissage hétérogènes d'aujourd'hui.

La finalité de cette modeste contribution est double : aider à mieux identifier la complexité qui caractérise la compétence de compréhension en lecture et fournir quelques propositions didactiques afin de remédier efficacement aux entraves rencontrées par les étudiants inscrits en FLE lors de cette activité. Nous espérons qu'au travers de cette étude, qui se base sur un échantillon infime, avoir pu apporter quelques éléments de réponses.

\section{Liste de références}

Al-Seghayer, K. (2005). The effects of verbal and spatial abilities on reading comprehension task performance in multimedia environments with respect to individual differences among learners. CALL-EJ Online, 7(1). Repéré à : http://www.callej.org/journal/7-1/Al-Seghayer.html

Ammouden, A. \& Ammouden, M. (2010). Le français à l'université et l'échelle des compétences du Cadre Européen Commun de Référence pour les Langues. Synergies Algérie 2010(9), 37-44.

Assude T. \& Loisy C. (2008). La dialectique acculturation/déculturation au cœur des systèmes de formation des enseignants aux TIC. Informations, Savoirs, Décisions et Médiations (ISDM), 2008(32) Repéré à : http://isdm.univ-tln.fr/PDF/isdm32/isdm32-assude.pdf

Baccino, T. (2004). La lecture électronique. Grenoble : Presses Universitaires de Grenoble.

Best, R. M., Row, M., Ozuru, Y. \& MacNamara, D. S. (2005). Deep-level comprehension of science text. The role of the reader and the text. Top Lang Disorder, 25(1), 65-83.

Bianco, M. (2015). Du langage oral à la compréhension de l'écrit. France : Presses universitaires de Grenoble.

Biggs, J. (1996). Enhancing teaching through constructive alignment. Higher Education, 1996(32), 347-364.

Boudechiche, N. (2008). Contributions à la didactique du texte expositif : Cas d'étudiants de filière scientifique. Annaba: Université Badji Mokhtar.

Cain, K. Oakhill, J. \& Bryant, P. (2000). Investigating the causes of reading comprehension failure: The comprehension-age match design. Reading and Writing: An Interdisciplinary Journal, 2000(12), 31-40. 
Cain, K. Oakhill, J. \& Bryant, P. (2004). Children's reading comprehension ability: concurrent prediction by working memory, verbal ability and component skills. Journal of Educational Psychology, 96(1), 3142.

Carliste, J. F. et Rice, M. S. (2002). Improving reading comprehension: Research-based principales and practices. Timonium, MD: York Press Inc.

Charlier, B., Deschryver. N. et Peraya, D. (2006). Apprendre en présence et à distance. Une définition des dispositifs hybrides. Distances et Savoirs, 4(4), 469-496.

Clark, R. E. (1994). Media Will Never Influence Learning. Educational Technology Research and Development, 42(2), 21-29.

Clarke, P. J., Truelove, E., Hulmes, C. \& Snowling, M. J. (2014). Developing Reading Comprehension. UK, West Sussex: John Wiley \& Sons.

Coiro, J. (2003). Reading comprehension on the Internet: Expanding our understanding of reading comprehension to encompass new literacies. The Reading Teacher, 2003(56), 453-458.

Dalton, B. \& Proctor, C. P. (2007). Reading as thinking: integrating strategy instruction in a universally designed digital literacy environment. Dans McNamara, D. S. (dir.), Reading comprehension strategies: theories, interventions, and technologies. (p. 421-439). Lawrence Erlbaum Assoc Inc: Mahwah, NJ. Repéré à : http://fliphtml5.com/xibl/ezxz/basic

Depover, C., Karsenti, T., Komis, V. (2007). Enseigner avec les technologies : favoriser les apprentissages, développer des compétences. Sainte-Foy : Presses de I'Université du Québec, ISBN 978-2-7605$1489-8,280$

Fan, Y. C. (2012). Peer collaboration for text comprehension among Taiwanese university learners. Arab World English Journal, 3(4), 113-133.

Farza, L. (2015). Impact d'une approche d'enseignement/apprentissage mixte sur les résultats des apprenants : cas d'un cours de bases de données. Spirale-E. Revue de Recherches en Education, Académie de Lille, 2015(55), 61-74. Repéré à : https://spirale-edu-revue.fr/spip.php?article1232

Ghorbani, M. R. \& Nezamoshari'e, M. N. (2012). Cooperative learning boosts EFL students' grammar achievement. Theory and Practice in Language Studies, 2(7), 1465-1471.

Irwin, J. W. (2007). Teaching reading comprehension processes. 3rd edition. Boston, MA : Pearson Allyn and Baconl.

Kadiyala M., Crynes, B. L. (2000). A review of literature on effectiveness of use of Information Technology in Education. Journal of engineering education, 89(2), 177-190.

Kangsepp, P. (2011). Impact of asking support questions on grades 4 and 7 students reading comprehension. Creative Education, 2(4). https://doi.org/10.4236/ce.2011.24054

Karsenti, T. (2004). Intégration des TIC en pédagogies universitaires : Bilan d'une enquête auprès de 700 formateurs du Québec (Canada). Actes du 21ème Congrès de l'Association Internationale de Pédagogie Universitaire 3-7 mai 2004. Marrakech, Maroc.

Kazuhiro, E. (2008). The effects of types of question on EFL learners' reading comprehension scores. (Thèse de doctorat). Philadelphia: Temple University. Repéré à : https://digital.library.temple.edu/digital/collection/p245801coll10/id/9182/rec/1

Khelfi. A, Zarrouk Ben Abid, S, Kadi Ksouri, L. (2018). Recherche-Action : Efficacité d'un dispositif d'enseignement hybride d'aide à la compréhension de texte produits en FLE. International Journal of Applied Research and Technology (IJARTech), 2018(1), 1-13.

Kherbache, A. (2008). Problématique de l'écriture et activités de scription : cas d'apprenants préparant une licence de français. Thèse de doctorat. Université d'Annaba.

Kherra, N. (2011). L'adaptation du CECRL aux besoins d'étudiants arabophones non spécialistes du français : cas des étudiants de l'école préparatoire d'architecture dans le contexte universitaire algérien. Synergies Europe, 2011(6), 85-98. 
Kintsch, W. (1998). Comprehension: A Paradigm for Cognition. NY: Cambridge University Press.

Lebrun, M. (2007). Quality towards an expected harmony: Pedagogy and technology speaking together about innovation. AACE Journal, 15(2), 115-130. Repéré à : http://www.editlib.org/p/21024

Lebrun, M. (2011). Impacts des TIC sur la qualité des apprentissages des étudiants et le développement professionnel des enseignants : vers une approche systémique. Revue des Sciences et Technologies de l'Information et de la Communication pour l'Education et la Formation (STICEF), 18(20).

Legros, D. (2006). (N)TIC et aides à la compréhension de textes et à la production d'écrits en L2 en contexte plurilingue et pluriculturel. Colloque Internationale Tice et Didactique des langues étrangères et maternelles.- Clermont 2. 14/15 sept. 2006. Université B. Pascal.

Legros, D. \& Baudet, S. (1996). Le rôle des modalisateurs épistémiques dans l'attribution de la vérité propositionnelle. International Journal of Psychology, 31(6), 235-253.

Legros, D. \& Crinon, J. (dir.). (2002). Psychologie des apprentissages et multimédia. Paris: Armand Colin.

Legros, D., Hoareau, Y., Boudechiche, N., Makhlouf, M. \& Gabsi, A. (2007). (N)TIC et aides à la compréhension et à la production de textes explicatifs en langue seconde.Vers une didactique cognitive du texte en contexte plurilingue et pluriculturel. ALSIC, 10(1), 33-49. Repéré à : https://journals.openedition.org/alsic/570

Legros, D., Pudelko, B., Crinon, J. \& Tricot, A. (2000). Les effets des systèmes et des outils multimédia sur la cognition, l'apprentissage et l'enseignement : Une articulation nécessaire entre la recherche théorique et la pratique de terrain. Education et Formation, 2000(56), 161-167.

Mangen, A., Walgermo, B. R. \& Brønnick, K. (2013). Reading linear texts on paper versus computer screen: Effects on reading comprehension. International Journal of Educational Research, 2013(58), 61-68.

Marin, B., Crinon, J., Legros, D. \& Avel, P. (2007). Lire un texte documentaire scientifique : Quels obstacles, quelles aides à la compréhension? Revue française de pédagogie, 2007(160), 119-131.

McNamara, D. S., Levinstein, I. B. \& Boonthum, C. (2004). iSTART: Interactive Strategy Trainer for Active Reading and Thinking. Behavioral Research Methods, Instruments, and Computers, 36(2), 222-233.

McNamara, D. S. \& Shapiro, A. M. (2005). Multimedia and hypermedia solutions for promoting metacognitive engagement, coherence and learning. Journal Educational Computing Research, 33(1), 1-29.

Momtaz, E. \& Garner, M. (2010). Does collaborative learning improve EFL students' reading comprehension? Journal of Linguistics and Language Teaching, 1(1), 15-36.

Morgan. G. (2003). Faculty use of course management systems. Research Study from the EDUCAUSE Center for Applied Research. Repéré à : https://www.researchgate.net/publication/242532338 Faculty Use of Course Management Systems

O'Reilly, T., Sinclair, G. P. \& McNamara, D. S. (2004). iSTART: A web-based reading strategy intervention that improves students' science comprehension. Dans D. G. Kinshuk Sampson \& P. Isaías (dir.), Proceedings of the IADIS International Conference Cognition and Exploratory Learning in Digital Age (p. 173-180). CELDA 2004. Lisbon, Portugal: IADIS Press.

Ouhaibia, B. (2010). L'effet d'un environnement informatisé sur l'apprentissage/développement d'une littératie en FLE. Synergies Algérie, 2010(9), 109-120.

Ouhaibia, B. (2016). L'effet de la réécriture collaborative sur la cohérence de textes narratifs en FLE. ANNABA : Université Badji Mokhtar.

Pan, C. Y. \& Wu, H. Y. (2013). The cooperative learning effects on english reading comprehension and learning motivation of EFL Freshmen. English Language Teaching, 6(5), 13-27.

Rekrak, L. (2016). Carte des connaissances et compréhension/ production d'un texte explicatif en classe de langue. Oran: Université d'Oran 2.

Sales, N. (2013). Flipping the Classroom: Revolutionising Legal Research Training. Legal Information Management, 13(04), 231-235. 
Sebane, M. (2008). L'effet de deux modalités de prise d'information (audition d'un CM vs lecture d'un polycopié) sur la réécriture d'un texte de spécialité en langue L2. Un enjeu pour la didactique de l'apprentissage en L2 et l'évaluation des compétences en producti. Synergies Algérie, 2008(2), 117-123.

Sebane, M. (2011). FOS / FOU : Quel « français » pour les étudiants algériens des filières scientifiques? Le Français sur Objectifs Universitaires, 375-380.

Snow, C. E. (2010). Academic language and the challenge of reading for learning about science. Science, 2010(328), 450-452. Repéré à : http://colabradio.mit.edu/wpcontent/uploads/2010/05/academiclanguage.pdf

Sperber, D. \& Wilson, D. (1989). La pertinence : communication et cognition. Paris: Minuit.

Tardif, J. (1996). Une condition incontournable aux promesses des NTIC en apprentissage : une pédagogie rigoureuse. Communication présentée au 14e colloque de l'AQUOPS. Québec. Repéré à : http://tecfalabs.unige.ch/mitic/articles/tardif 1996 une condition incontournable aux promesses de $\mathrm{s}$ ntic en apprentissage une pedagogie rigoureuse.pdf

Tiberghien, G. (1997). La mémoire oubliée. Liège: Mardaga, 206 p.

Tierney, R. (2009). The agency and artistry of meaning makers within and across digital spaces. Dans S. E. Israel, Handbook of research on reading comprehension (p. 261-288). New York: Routledge.

Tricot, A., Plégat-Soutjis, F., Camps, J.-F., Amiel, A., Lutz, G. et Morcillo, A. (2003). Utilité, utilisabilité, acceptabilité : interpréter les relations entre trois dimensions de l'évaluation des EIAH. Dans Actes du colloque Environnements Informatiques pour l'Apprentissage Humain. Strasbourg, France, p. 391-402.

Van Dijk, T.A. \& Kintsch, W. (1983). Strategies of discourse comprehension. New York: Academic Press.

Vidal-Abarca, E., Reyes, H., Gilabert, R., Calpe, J., Soria, E. \& Graesser, A. C. (2002). ETAT: Expository Text Analysis Tool. Behavior Research Methods, Instruments, and Computers, 2002(34), 93-107.

Wright, S., Fugett, A. \& Caputa, F. (2013). Using E-readers and internet resources to support comprehension. Educational Technology \& Society, 16(1), 367-379. Repéré à : https://www.researchgate.net/publication/282499458 Using Ereaders and internet resources to support comprehension

Yee, N. \& Mclntyre, L. J. (2013). Understanding reading: A model of meaningful reading. Education Matters, 1(1), 53-79.

Zuo, W. (2011). The effects of cooperative learning on improving college students' reading comprehension. Theory and Practice in Language Studies, 1(8), 986-989. 